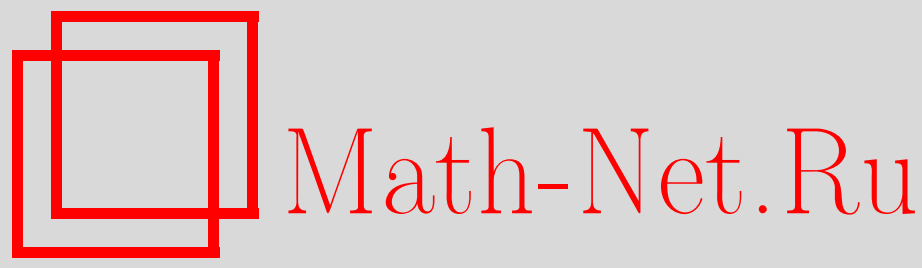

Н. А. Перязев, И. К. Шаранхаев, Теория Галуа для клонов и суперклонов, Дискрет. матем., 2015, том 27, выпуск 4, 79-93

DOI: https://doi.org/10.4213/dm1349

Использование Общероссийского математического портала Math-Net.Ru подразумевает, что вы прочитали и согласны с пользовательским соглашением http://www . mathnet.ru/rus/agreement

Параметры загрузки:

IP : 34.227 .88 .159

26 апреля 2023 г., 12:51:23

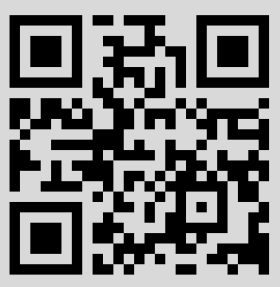




\title{
Теория Галуа для клонов и суперклонов
}

\author{
() 2015 г. Н. А. Перязев*, И. К. Шаранхаев**
}

Рассматриваются клоны (замкнутые множества операций, содержащие все проекции) и суперклоны над конечными множествами. Следуя А.И. Мальцеву, клон можно рассматривать как алгебру. Если в такой алгебре основное множество заменить на множество мультиопераций и добавить операцию разрешимости простейшего уравнения, то получим алгебру, называемую суперклоном. В работе устанавливается связь Галуа между клонами и суперклонами.

Работа выполнена при поддержке РФФИ, проект 12-01-000351а.

Ключевые слова: клон, суперклон, операция, мультиоперация, суперпозиция

\section{1. Введение}

Одним из первых систематические исследования функциональных систем конечных функций начал С.В.Яблонский $[1,2]$. Алгебраический подход к изучению функциональных систем впервые был предложен А.И. Мальцевым [3]. Среди алгебр функций наибольшее распространение получили клоны - алгебры операций, замкнутые относительно суперпозиции и содержащие операции проекций $[4,5]$. В [6, 7] устанавливается связь Галуа для клонов и ко-клонов - алгебр отношений.

Рассматривая алгебры над множествами мультиопераций с одной дополнительной операцией разрешимости простейшего уравнения, получаем алгебру, называемую суперклоном [8]. Так как решетки суперклонов и ко-клонов изоморфны [9], то, возможно, существует связь Галуа для клонов и суперклонов. В данной работе эта связь устанавливается, доказательство теорем основано на идеях из [6]. Результаты работы анонсированы в $[10,11]$.

\section{2. Клоны и суперклоны}

Пусть $A$ - произвольное фиксированное множество. Отображение из $A^{n}$ в $A$ называется $n$-местной операцией на $A$ (будем допускать случай $n=0$ ). Множество всех

* Место работы: Санкт-Петербургский государственный электротехнический университет, e-mail: nikolai.baikal@gmail.com

** Место работы: Бурятский государственный университет, e-mail: goran5@mail.ru 
$n$-местных операций на $A$ обозначаем через $P_{A}^{n}$, а множество всех операций на $A$ через

$$
P_{A}=\bigcup_{n \geqslant 0} P_{A}^{n} .
$$

Пусть $K \subseteq P_{A}$. Алгебра $\mathfrak{K}=<K ; *, \zeta, \tau, \Delta, \varepsilon>$ типа $\langle 2,1,1,1,0\rangle$ с ниже определенными операциями подстановки $(f * g)$, циклической перестановки аргументов $(\zeta f)$, транспозиции аргументов $(\tau f)$, отождествления аргументов $(\Delta f)$ и операцией $\varepsilon$, выделяющей операцию проекции по первому аргументу $e_{1}^{2} \in P_{A}^{2}$, называется клоном над $A$.

Если $f \in K \cap P_{A}^{n}$ и $g \in K \cap P_{A}^{m}$, то

$(f * g)\left(a_{1}, \ldots, a_{n+m-1}\right)=f\left(g\left(a_{1}, \ldots, a_{m}\right), a_{m+1}, \ldots, a_{n+m-1}\right)$ при $n \geqslant 1$;

$(f * g)\left(a_{1}, \ldots, a_{m}\right)=f()$ при $n=0$;

$(\zeta f)\left(a_{1}, \ldots, a_{n}\right)=f\left(a_{2}, \ldots, a_{n}, a_{1}\right)$ при $n>1$ и $(\zeta f)=f$ при $n \leqslant 1$;

$(\tau f)\left(a_{1}, \ldots, a_{n}\right)=f\left(a_{2}, a_{1}, a_{3}, \ldots, a_{n}\right)$ при $n>1$ и $(\tau f)=f$ при $n \leqslant 1$;

$(\Delta f)\left(a_{1}, \ldots, a_{n-1}\right)=f\left(a_{1}, a_{1}, a_{2}, \ldots, a_{n-1}\right)$ при $n>1$,

$(\Delta f)()=b$, если $f(a)=b$ для всех $a \in A$, иначе $(\Delta f)=f$ при $n=1$,

$(\Delta f)=f$ при $n=0$;

$\varepsilon=e_{1}^{2}$, где $e_{1}^{2}\left(a_{1}, a_{2}\right)=a_{1}$.

Пусть $B(A)$ - множество всех подмножеств $A$, в том числе $\varnothing$. Отображение из $A^{n}$ в $B(A)$ называется $n$-местной мультиоперацией на $A$ (будем допускать случай $n=0)$. Для множества всех $n$-местных мультиопераций на $A$ используем обозначение $M_{A}^{n}$, а для множества всех мультиопераций на $A$ обозначение

$$
M_{A}=\bigcup_{n \geqslant 0} M_{A}^{n}
$$

Пусть $R \subseteq M_{A}$. Алгебра $\mathfrak{R}=<R ; *, \zeta, \tau, \Delta, \mu, \varepsilon>$ типа $\langle 2,1,1,1,1,0\rangle$ с ниже определенными операциями подстановки $(f * g)$, циклической перестановки аргументов $(\zeta f)$, транспозиции аргументов $(\tau f)$, отождествления аргументов $(\Delta f)$, разрешимости $(\mu f)$ и операцией $\varepsilon$, выделяющей бинарную мультиоперацию проекции по первому аргументу, называется суперклоном над $A$.

Если $f \in R \cap M_{A}^{n}$ и $g \in R \cap M_{A}^{m}$, то

$(f * g)\left(a_{1}, \ldots, a_{n+m-1}\right)=\left\{a \mid\right.$ существует такой $a_{0} \in g\left(a_{1}, \ldots, a_{m}\right)$, что

$\left.a \in f\left(a_{0}, a_{m+1}, \ldots, a_{m+n-1}\right)\right\}$ при $n \geqslant 1$,

$(f * g)\left(a_{1}, \ldots, a_{m}\right)=f()$, если $g\left(a_{1}, \ldots, a_{m}\right) \neq \varnothing$ и

$(f * g)\left(a_{1}, \ldots, a_{m}\right)=\varnothing$, если $g\left(a_{1}, \ldots, a_{m}\right)=\varnothing$ при $n=0$;

$(\zeta f)\left(a_{1}, \ldots, a_{n}\right)=f\left(a_{2}, \ldots, a_{n}, a_{1}\right)$ при $n>1,(\zeta f)=f$ при $n \leqslant 1$;

$(\tau f)\left(a_{1}, \ldots, a_{n}\right)=f\left(a_{2}, a_{1}, a_{3}, \ldots, a_{n}\right)$ при $n>1,(\tau f)=f$ при $n \leqslant 1$;

$(\Delta f)\left(a_{1}, \ldots, a_{n-1}\right)=f\left(a_{1}, a_{1}, a_{2}, \ldots, a_{n-1}\right)$ при $n>1$,

$(\Delta f)()=\{a \mid a \in f(a)\}$ при $n=1,(\Delta f)=f$ при $n=0$;

$(\mu f)\left(a_{1}, \ldots, a_{n}\right)=\left\{a \mid a_{1} \in f\left(a, a_{2}, \ldots, a_{n}\right)\right\}$, при $n \geqslant 1$,

$(\mu f)()=\varnothing$ при $n=0$;

$\varepsilon=e_{1}^{2}$, где $e_{1}^{2}\left(a_{1}, a_{2}\right)=\left\{a_{1}\right\}$.

Мультиоперации, на всех наборах равные одноэлементным множествам, ничем не отличаются от операций. Поэтому такие мультиоперации будем отождествлять с соответствующими им операциями. 
Мощность множества $A$ называется рангом клона (суперклона). В дальнейшем будем рассматривать клоны и суперклоны только конечных рангов.

Будем говорить, что множество операций (мультиопераций) образует клон (суперклон), если это множество с определенными выше операциями является клоном (суперклоном).

\section{3. Суперпозиция мультиопераций и полутождество суперассоциативности}

Используя главные операции суперклона, можно определить следующие частичные операции в суперклоне:

1) проекции по $i$-му аргументу $e_{i}^{n}\left(a_{1}, \ldots, a_{n}\right)=\left\{a_{i}\right\}$;

2 ) отождествления $j$-го аргумента с $i$-м аргументом

$$
\left(\Delta_{i, j} f\right)\left(a_{1}, \ldots, a_{j-1}, a_{j+1}, \ldots, a_{n}\right)=f\left(a_{1}, \ldots, a_{j-1}, a_{i}, a_{j+1}, \ldots, a_{n}\right)
$$

3) подстановки на место $i$-го аргумента

$$
\left(f *_{i} g\right)\left(a_{1}, \ldots, a_{n+m-1}\right)=\bigcup_{b \in g\left(a_{i}, \ldots, a_{i+m-1}\right)} f\left(a_{1}, \ldots, a_{i-1}, b, a_{i+m}, \ldots, a_{n+m-1}\right) ;
$$

4) суперпозиции для $f \in M_{A}^{n}$ и $f_{i} \in M_{A}^{m}(i=1, \ldots, n)$

$$
f *\left(f_{1}, \ldots, f_{n}\right)\left(a_{1}, \ldots, a_{m}\right)=\bigcup_{b_{i} \in f_{i}\left(a_{1}, \ldots, a_{m}\right)} f\left(b_{1}, \ldots, b_{n}\right) .
$$

Если $f, f_{1}, \ldots, f_{n}$ являются операциями, то получается общепринятое понятие суперпозиции операций.

Как установил А.И. Мальцев [3], множество операций $K$ образует клон тогда и только тогда, когда $K$ содержит все проекции $e_{i}^{n}$ и замкнуто относительно суперпозиции. Аналогично, нетрудно показать, что множество мультиопераций $R$ образует суперклон тогда и только тогда, когда $R$ содержит все проекции $e_{i}^{n}$ и замкнуто относительно суперпозиции и операции разрешимости.

Лемма 1. Для суперпозиции мультиопераций выполняется полутождество суперассоииативности

$$
\left(g_{0} *\left(g_{1}, \ldots, g_{s}\right)\right) *\left(h_{1}, \ldots, h_{m}\right) \subseteq g_{0} *\left(g_{1} *\left(h_{1}, \ldots, h_{m}\right), \ldots, g_{s} *\left(h_{1}, \ldots, h_{m}\right)\right) .
$$

Если $h_{1}, \ldots, h_{m}$ являются операчиями, то выполняется тождество суперассоциативности

$$
\left(g_{0} *\left(g_{1}, \ldots, g_{s}\right)\right) *\left(h_{1}, \ldots, h_{m}\right)=g_{0} *\left(g_{1} *\left(h_{1}, \ldots, h_{m}\right), \ldots, g_{s} *\left(h_{1}, \ldots, h_{m}\right)\right) .
$$

Доказательство. Пусть $a_{1}, \ldots, a_{n}$ - произвольный набор элементов из $A$. Если

$$
b \in\left(g_{0} *\left(g_{1}, \ldots, g_{s}\right)\right) *\left(h_{1}, \ldots, h_{m}\right)\left(a_{1}, \ldots, a_{n}\right),
$$


TO

$$
b \in g_{0} *\left(g_{1}, \ldots, g_{s}\right)\left(c_{1}, \ldots, c_{m}\right)
$$

для некоторых $c_{i} \in h_{i}\left(a_{1}, \ldots, a_{n}\right), i=1, \ldots, m$. А значит, $b \in g_{0}\left(d_{1}, \ldots, d_{s}\right)$ для некоторых $d_{j} \in g_{j}\left(c_{1}, \ldots, c_{m}\right), j=1, \ldots, s$. Отсюда получаем, что

$$
d_{j} \in g_{j} *\left(h_{1}, \ldots, h_{m}\right)\left(a_{1}, \ldots, a_{n}\right), j=1, \ldots, s .
$$

Учитывая, $b \in g_{0}\left(d_{1}, \ldots, d_{s}\right)$, получаем

$$
b \in g_{0} *\left(g_{1} *\left(h_{1}, \ldots, h_{m}\right), \ldots, g_{s} *\left(h_{1}, \ldots, h_{m}\right)\right)\left(a_{1}, \ldots, a_{n}\right),
$$

что и требовалось.

Если $h_{1}, \ldots, h_{m}$ являются операциями, то существуют такие $c_{i}$, что

$$
c_{i}=h_{i}\left(a_{1}, \ldots, a_{n}\right), i=1, \ldots, m \text {. }
$$

Пусть

$$
b \in g_{0} *\left(g_{1} *\left(h_{1}, \ldots, h_{m}\right), \ldots, g_{s} *\left(h_{1}, \ldots, h_{m}\right)\right)\left(a_{1}, \ldots, a_{n}\right) .
$$

Тогда $b \in g_{0}\left(d_{1}, \ldots, d_{s}\right)$ для $d_{j} \in g_{j} *\left(h_{1}, \ldots, h_{m}\right)\left(a_{1}, \ldots, a_{n}\right), j=1, \ldots, s$. А значит, $d_{j} \in g_{j}\left(c_{1}, \ldots, c_{m}\right)$, где $c_{i}=h_{i}\left(a_{1}, \ldots, a_{n}\right), i=1, \ldots, m$. Отсюда получаем $d_{j} \in$ $g_{j}\left(h_{1}\left(a_{1}, \ldots, a_{n}\right), \ldots, h_{m}\left(a_{1}, \ldots, a_{n}\right)\right), j=1, \ldots, s$. Теперь, учитывая $b \in g_{0}\left(d_{1}, \ldots, d_{s}\right)$, находим:

$$
b \in g_{0} *\left(g_{1}, \ldots, g_{s}\right)\left(h_{1}\left(a_{1}, \ldots, a_{n}\right), \ldots, h_{m}\left(a_{1}, \ldots, a_{n}\right)\right) .
$$

Поэтому $b \in\left(g_{0} *\left(g_{1}, \ldots, g_{s}\right)\right) *\left(h_{1}, \ldots, h_{m}\right)\left(a_{1}, \ldots, a_{n}\right)$. Этим доказали обратное включение, а значит, и равенство.

Заметим, что в случае, когда $h_{1}, \ldots, h_{m}$ не являются операциями, легко показать, что равенство может не выполняться [9].

\section{4. Стабилизаторы и нормализаторы}

Если для любых элементов $a_{1}^{1}, \ldots, a_{m}^{1}, \ldots, a_{1}^{n}, \ldots, a_{m}^{n}$ из $A$ выполняется включение

$$
f *\left(g\left(a_{1}^{1}, \ldots, a_{m}^{1}\right), \ldots, g\left(a_{1}^{n}, \ldots, a_{m}^{n}\right)\right) \subseteq g *\left(f\left(a_{1}^{1}, \ldots, a_{1}^{n}\right), \ldots, f\left(a_{m}^{1}, \ldots, a_{m}^{n}\right)\right),
$$

то говорим, что для мультиопераций $f$ и $g$ верно полутождество перестановочноcти, а также, что $f$ стабильна относительно $g$, а $g$ нормальна относительно $f$.

Если $g \in M_{A}$, то множество $S(g)=\left\{f \mid f \in P_{A}\right.$ и $f$ стабильна относительно $\left.g\right\}$ называется стабилизатором $g$.

Если $f \in P_{A}$, то множество $N(f)=\left\{g \mid g \in M_{A}\right.$ и $g$ нормальна относительно $\left.f\right\}$ называется нормализатором $f$.

Если $R \subseteq M_{A}$, то $S(R)=\bigcap_{g \in R} S(g)-$ стабилизатор множества мультиопераций $R$.

Если $K \subseteq P_{A}$, то $N(K)=\bigcap_{f \in K} N(f)-$ нормализатор множества операций $K$.

Предложение 1. а) Стабилизатор $S(R)$ образует клон для любого $R \subseteq M_{A}$; б) Нормализатор $N(K)$ образует суперклон для любого $K \subseteq P_{A}$. 
Доказательство. а) Покажем, что $S(R)$ содержит проекции и замкнуто относительно суперпозиции. Пусть $g \in R \cap M_{A}^{m}$ и

$$
\begin{gathered}
e_{i}^{n} *\left(g\left(a_{1}^{1}, \ldots, a_{m}^{1}\right), \ldots, g\left(a_{1}^{n}, \ldots, a_{m}^{n}\right)\right) \subseteq \\
\subseteq g\left(a_{1}^{i}, \ldots, a_{m}^{i}\right)=g *\left(e_{i}^{n}\left(a_{1}^{1}, \ldots, a_{1}^{n}\right), \ldots, e_{i}^{n}\left(a_{m}^{1}, \ldots, a_{m}^{n}\right)\right) .
\end{gathered}
$$

Получили, что $e_{i}^{n} \in S(R)$.

Пусть $f_{0}, f_{1}, \ldots, f_{s} \in S(R)$. Тогда, используя полутождество суперассоциативности (лемма 1), находим:

$$
\begin{aligned}
& \left(f_{0} *\left(f_{1}, \ldots, f_{s}\right)\right) *\left(g\left(a_{1}^{1}, \ldots, a_{m}^{1}\right), \ldots, g\left(a_{1}^{n}, \ldots, a_{m}^{n}\right)\right) \subseteq \\
& \subseteq f_{0} *\left(f_{1} *\left(g\left(a_{1}^{1}, \ldots, a_{m}^{1}\right), \ldots, g\left(a_{1}^{n}, \ldots, a_{m}^{n}\right)\right), \ldots,\right. \\
& \left.\ldots, f_{s} *\left(g\left(a_{1}^{1}, \ldots, a_{m}^{1}\right), \ldots, g\left(a_{1}^{n}, \ldots, a_{m}^{n}\right)\right)\right) \subseteq \\
& \subseteq f_{0} *\left(g *\left(f_{1}\left(a_{1}^{1}, \ldots, a_{1}^{n}\right), \ldots, f_{1}\left(a_{m}^{1}, \ldots, a_{m}^{n}\right)\right), \ldots,\right. \\
& \left.\ldots, g *\left(f_{s}\left(a_{1}^{1}, \ldots, a_{1}^{n}\right), \ldots, f_{s}\left(a_{m}^{1}, \ldots, a_{m}^{n}\right)\right)\right) \subseteq \\
& \subseteq g *\left(f_{0} *\left(f_{1}\left(a_{1}^{1}, \ldots, a_{1}^{n}\right), \ldots, f_{s}\left(a_{1}^{1}, \ldots, a_{1}^{n}\right)\right), \ldots,\right. \\
& \left.\ldots, f_{0} *\left(f_{1}\left(a_{m}^{1}, \ldots, a_{m}^{n}\right), \ldots, f_{s}\left(a_{m}^{1}, \ldots, a_{m}^{n}\right)\right)\right)= \\
& =g *\left(f_{0} *\left(f_{1}, \ldots, f_{s}\right)\left(a_{1}^{1}, \ldots, a_{1}^{n}\right), \ldots, f_{0} *\left(f_{1}, \ldots, f_{s}\right)\left(a_{m}^{1}, \ldots, a_{m}^{n}\right)\right) \text {. }
\end{aligned}
$$

Получили $f_{0} *\left(f_{1}, \ldots, f_{s}\right) \in S(R)$.

б) Покажем, что $N(K)$ содержит проекции и замкнуто относительно суперпозиции и операции разрешимости. Пусть $f \in K \cap P_{A}^{n}$. Тогда

$$
\begin{gathered}
f *\left(e_{i}^{m}\left(a_{1}^{1}, \ldots, a_{m}^{1}\right), \ldots, e_{i}^{m}\left(a_{1}^{n}, \ldots, a_{m}^{n}\right)\right)= \\
=f\left(a_{i}^{1}, \ldots, a_{i}^{n}\right)=e_{i}^{m} *\left(f\left(a_{1}^{1}, \ldots, a_{1}^{n}\right), \ldots, f\left(a_{m}^{1}, \ldots, a_{m}^{n}\right)\right) .
\end{gathered}
$$

Получили $e_{i}^{m} \in N(K)$.

Пусть $g_{0}, g_{1}, \ldots, g_{s} \in N(K)$. Тогда

$$
a \in f *\left(g_{0} *\left(g_{1}, \ldots, g_{s}\right)\left(a_{1}^{1}, \ldots, a_{m}^{1}\right), \ldots, g_{0} *\left(g_{1}, \ldots, g_{s}\right)\left(a_{1}^{n}, \ldots, a_{m}^{n}\right)\right) .
$$

А значит, $a=f\left(b_{1}, \ldots, b_{n}\right)$, где $b_{i} \in g_{0} *\left(g_{1}, \ldots, g_{s}\right)\left(a_{1}^{i}, \ldots, a_{m}^{i}\right), i=1, \ldots, n$. Поэтому $b_{i} \in g_{0}\left(c_{1}^{i}, \ldots, c_{s}^{i}\right)$, где $c_{j}^{i} \in g_{j}\left(a_{1}^{i}, \ldots, a_{m}^{i}\right), \quad j=1, \ldots, s$. Отсюда $f\left(c_{j}^{1}, \ldots, c_{j}^{n}\right) \in$ $f *\left(g_{j}\left(a_{1}^{1}, \ldots, a_{m}^{1}\right), \ldots, g_{j}\left(a_{1}^{n}, \ldots, a_{m}^{n}\right)\right)$. В силу того, что $g_{j} \in N(K)$, получаем $f\left(c_{j}^{1}, \ldots, c_{j}^{n}\right) \in g_{j} *\left(f\left(a_{1}^{1}, \ldots, a_{1}^{n}\right), \ldots, f\left(a_{m}^{1}, \ldots, a_{m}^{n}\right)\right)$. Отсюда

$$
\begin{gathered}
g_{0} *\left(f\left(c_{1}^{1}, \ldots, c_{1}^{n}\right), \ldots, f\left(c_{s}^{1}, \ldots, c_{s}^{n}\right)\right) \subseteq g_{0} *\left(g _ { 1 } * \left(f\left(a_{1}^{1}, \ldots, a_{1}^{n}\right), \ldots,\right.\right. \\
\left.\left.\ldots, f\left(a_{m}^{1}, \ldots, a_{m}^{n}\right)\right), \ldots, g_{s} *\left(f\left(a_{1}^{1}, \ldots, a_{1}^{n}\right), \ldots, f\left(a_{m}^{1}, \ldots, a_{m}^{n}\right)\right)\right) .
\end{gathered}
$$

Tак как $g_{0} \in N(K)$, то

$$
f *\left(g_{0}\left(c_{1}^{1}, \ldots, c_{s}^{1}\right), \ldots, g_{0}\left(c_{1}^{n}, \ldots, c_{s}^{n}\right)\right) \subseteq g_{0} *\left(f\left(c_{1}^{1}, \ldots, c_{1}^{n}\right), \ldots, f\left(c_{s}^{1}, \ldots, c_{s}^{n}\right)\right) .
$$


Так как $a=f\left(b_{1}, \ldots, b_{n}\right)$ и $b_{i} \in g_{0}\left(c_{1}^{i}, \ldots, c_{s}^{i}\right), i=1, \ldots, n$, то

$$
\begin{gathered}
a \in g_{0} *\left(g_{1} *\left(f\left(a_{1}^{1}, \ldots, a_{1}^{n}\right), \ldots, f\left(a_{m}^{1}, \ldots, a_{m}^{n}\right)\right), \ldots,\right. \\
\left.\ldots, g_{s} *\left(f\left(a_{1}^{1}, \ldots, a_{1}^{n}\right), \ldots, f\left(a_{m}^{1}, \ldots, a_{m}^{n}\right)\right)\right) .
\end{gathered}
$$

Применим тождество суперассоциативности, учитывая, что $f$ является операцией:

$$
a \in\left(g_{0} *\left(g_{1}, \ldots, g_{s}\right)\right) *\left(f\left(a_{1}^{1}, \ldots, a_{1}^{n}\right), \ldots, f\left(a_{m}^{1}, \ldots, a_{m}^{n}\right)\right) .
$$

Таким образом, $g_{0} *\left(g_{1}, \ldots, g_{s}\right) \in N(K)$.

Пусть $g \in N(K)$ и $a \in f *\left((\mu g)\left(a_{1}^{1}, \ldots, a_{m}^{1}\right), \ldots,(\mu g)\left(a_{1}^{n}, \ldots, a_{m}^{n}\right)\right)$. Значит, $a=$ $f\left(b_{1}, \ldots, b_{n}\right)$, где $b_{i} \in(\mu g)\left(a_{1}^{i}, \ldots, a_{m}^{i}\right), i=1, \ldots, n$. Отсюда

$$
a_{1}^{i} \in g\left(b_{i}, a_{2}^{i}, \ldots, a_{m}^{i}\right), i=1, \ldots, n .
$$

Поэтому

$$
f\left(a_{1}^{1}, \ldots, a_{1}^{n}\right) \in f *\left(g\left(b_{1}, a_{2}^{1}, \ldots, a_{m}^{1}\right), \ldots, g\left(b_{n}, a_{2}^{n}, \ldots, a_{m}^{n}\right)\right) .
$$

Taк как $g \in N(K)$, то

$$
f\left(a_{1}^{1}, \ldots, a_{1}^{n}\right) \in g *\left(f\left(b_{1}, \ldots, b_{n}\right), f\left(a_{2}^{1}, \ldots, a_{2}^{n}\right), \ldots, f\left(a_{m}^{1}, \ldots, a_{m}^{n}\right)\right) .
$$

Отсюда $f\left(b_{1}, \ldots, b_{n}\right) \in(\mu g) *\left(f\left(a_{1}^{1}, \ldots, a_{1}^{n}\right), \ldots, f\left(a_{m}^{1}, \ldots, a_{m}^{n}\right)\right)$. Поэтому

$$
a \in(\mu g) *\left(f\left(a_{1}^{1}, \ldots, a_{1}^{n}\right), \ldots, f\left(a_{m}^{1}, \ldots, a_{m}^{n}\right)\right) .
$$

Получили $(\mu g) \in N(K)$.

\section{5. Алгебраическое замыкание и замыкание Галуа в клонах}

Стандартным образом определяется алгебраическое замыкание множества операций $K$, а именно $[K]=\bigcap_{K \subseteq K_{i}} K_{i}$, где пересечение берется по всевозможным клонам $K_{i}$, содержащим $K$.

Замыканием Галуа множества операций $K$ называется клон $S(N(K))$.

Теорема 1. В клонах алгебрачческие замыкания совпадают с замыканиями Галуа: $[K]=S(N(K))$.

Доказательству теоремы предпошлем вспомогательное утверждение.

Пусть $|A|=k$ и $h_{1}, \ldots, h_{s}$ - все операции размерности $n$ из клона $[K]$. Тогда $n$-универсальной для $[K]$ называется мультиоперация $h$, определенная следующим образом:

$$
h\left(b_{1}, \ldots, b_{t}\right)=\bigcup_{i=1}^{s}\left(\bigcup_{h_{i}(\widehat{1})=b_{1}, \ldots, h_{i}(\widehat{t})=b_{t}}\left\{h_{i}(\widehat{t+1})\right\}\right),
$$

где $t=k^{n}-1$ и $\widehat{1}, \ldots, \widehat{t+1}-$ все наборы элементов из $A$ длины $n$ в фиксированном порядке. 
Лемма 2. Пусть $h$ является п-универсальной мультиоперачией для клона $[K]$. Тогда $h \in N(K)$.

Доказательство. Необходимо доказать для любой $g \in K^{m}$ выполнение включения $g *\left(h\left(a_{1}^{1}, \ldots, a_{t}^{1}\right), \ldots, h\left(a_{1}^{m}, \ldots, a_{t}^{m}\right)\right) \subseteq h *\left(g\left(a_{1}^{1}, \ldots, a_{1}^{m}\right), \ldots, g\left(a_{t}^{1}, \ldots, a_{t}^{m}\right)\right)$.

Пусть $b \in g *\left(h\left(a_{1}^{1}, \ldots, a_{t}^{1}\right), \ldots, h\left(a_{1}^{m}, \ldots, a_{t}^{m}\right)\right)$. Тогда

$$
b=g *\left(h_{i_{1}}(\widehat{t+1}), \ldots, h_{i_{m}}(\widehat{t+1})\right),
$$

где

$$
\begin{gathered}
a_{1}^{1}=h_{i_{1}}(\widehat{1}), \cdots, a_{1}^{m}=h_{i_{m}}(\widehat{1}), \\
\vdots \\
a_{t}^{1}=h_{i_{1}}(\widehat{t}), \cdots, a_{t}^{m}=h_{i_{m}}(\widehat{t}) .
\end{gathered}
$$

Значит,

$$
\begin{gathered}
g\left(a_{1}^{1}, \ldots, a_{1}^{m}\right)=g *\left(h_{i_{1}}(\widehat{1}), \cdots, h_{i_{m}}(\widehat{1})\right), \\
\vdots \\
g\left(a_{t}^{1}, \ldots, a_{t}^{m}\right)=g *\left(h_{i_{1}}(\widehat{t}), \cdots, h_{i_{m}}(\widehat{t})\right) .
\end{gathered}
$$

Так как $g *\left(h_{i_{1}}, \cdots, h_{i_{m}}\right)$ является $n$-местной операцией из $[K]$, то она совпадает с некоторой операцией $h_{j}$. Отсюда получаем

$$
\begin{gathered}
g\left(a_{1}^{1}, \ldots, a_{1}^{m}\right)=h_{j}(\widehat{1}), \\
\vdots \\
g\left(a_{t}^{1}, \ldots, a_{t}^{m}\right)=h_{j}(\widehat{t}) .
\end{gathered}
$$

А в силу того, что $b=h_{j}(\widehat{t+1})$, по определению $h$ выполняется включение

$$
b \in h *\left(g\left(a_{1}^{1}, \ldots, a_{1}^{m}\right), \ldots, g\left(a_{t}^{1}, \ldots, a_{t}^{m}\right)\right) .
$$

Доказательство теоремы 1. Покажем сначала, что $[K] \subseteq S(N(K))$.

Пусть $f \in K$. Тогда $f$ стабильна относительно всех $g \in N(K)$, а значит, $f \in S(N(K))$. Следовательно, $K \subseteq S(N(K))$ и $[K] \subseteq[S(N(K))]$. Так как по предложению 1 любой стабилизатор является клоном, то

$$
[S(N(K))]=S(N(K))
$$

Теперь покажем, что $S(N(K)) \subseteq[K]$.

Пусть $f \in S(N(K))$, размерность $f$ равна $n$ и $h_{1}, \ldots, h_{s}$ - все операции размерности $n$ из клона $[K]$. По лемме 2 мультиоперация $h$, являющаяся $n$-универсальной для $[K]$, принадлежит $N(K)$.

Так как $f \in S(N(K))$ и $h \in N(K)$, то для любых $a_{1}^{1}, \ldots, a_{t}^{1}, \ldots, a_{1}^{n}, \ldots, a_{t}^{n}$

$$
f *\left(h\left(a_{1}^{1}, \ldots, a_{t}^{1}\right), \ldots, h\left(a_{1}^{n}, \ldots, a_{t}^{n}\right)\right) \subseteq h *\left(f\left(a_{1}^{1}, \ldots, a_{1}^{n}\right), \ldots, f\left(a_{t}^{1}, \ldots, a_{t}^{n}\right)\right) .
$$


Возьмем в качестве наборов $\left(a_{1}^{1}, \ldots, a_{1}^{n}\right), \ldots,\left(a_{t}^{1}, \ldots, a_{t}^{n}\right)$ соответственно наборы $\widehat{1}, \ldots, \widehat{t}$. Так как $n$-местные проекции содержатся среди $h_{1}, \ldots, h_{s}$, то

$$
\widehat{t+1} \in\left\{\left(c_{1}, \ldots, c_{n}\right) \mid c_{1} \in h\left(a_{1}^{1}, \ldots, a_{t}^{1}\right), \ldots, c_{n} \in h\left(a_{1}^{n}, \ldots, a_{t}^{n}\right)\right\} .
$$

Поэтому

$$
f(\widehat{t+1}) \in h(f(\widehat{1}), \ldots, f(\widehat{t})) .
$$

Тогда по определению $h$ существует такая операция $h_{j}$, что

$$
h_{j}(\widehat{1})=f(\widehat{1}), \ldots, h_{j}(\widehat{t})=f(\widehat{t}) \text { и } h_{j}(\widehat{t+1})=f(\widehat{t+1}) .
$$

Таким образом $f=h_{j}$, а значит, $f \in[K]$. Теорема доказана.

\section{6. Алгебраическое замыкание и замыкание Галуа в суперклонах}

Аналогично клонам, в суперклонах определяется алгебраическое замыкание множества мультиопераций $R$ как $\langle R\rangle=\bigcap_{R \subseteq R_{i}} R_{i}$, где $R_{i}$ являются всевозможными суперклонами, и замыкание Галуа как $N(S(R))$.

Теорема 2. В суперклонах алгебраические замыкания совпадают с замыканиями Галуа: $\langle R\rangle=N(S(R))$.

Сначала приведем несколько вспомогательных понятий и утверждений.

Каждую операцию $g \in M_{A}^{m}$, определенную хотя бы на одном наборе (т. е. должен существовать набор, на котором $g$ не равна $\varnothing)$, можно однозначно с точностью до перестановки столбцов представить в виде матрицы $X_{g}$, элементы которой принадлежат $A$ и в которой нет одинаковых столбцов, следующим образом: $\left(x_{1}, \ldots, x_{m}, y\right)$ является столбцом матрицы $X_{g}$ тогда и только тогда, когда $y \in g\left(x_{1}, \ldots, x_{m}\right)$. Отметим, что матрица $A$ состоит из $(m+1)$ строк и число столбцов в ней не может превосходить $2^{m+1}$.

Для произвольного отношения эквивалентности $\sim$ на множестве $\{1, \ldots, m+1\}$ определим мультиоперацию $d$ через представляющую ее матрицу

$$
\left(\begin{array}{llll}
a_{1}^{1} & a_{1}^{2} & \ldots & a_{1}^{k} \\
a_{2}^{1} & a_{2}^{2} & \ldots & a_{2}^{k} \\
\ldots & \ldots & \ldots & \ldots \\
a_{m+1}^{1} & a_{m+1}^{2} & \ldots & a_{m+1}^{k}
\end{array}\right)
$$

для элементов этой матрицы из $i \sim j$ следует, что $a_{i}^{s}=a_{j}^{s}$ для любого $s \in\{1, \ldots, k\}$. Так определенные мультиоперации назовем диагональными.

Пусть $\langle R\rangle$ - суперклон и $g \in\langle R\rangle$ имеет размерность $m$. Далее мы опишем действия над матрицей $X_{g}$, в результате которых получаются матрицы мультиопераций, которые выражаются через $g$ и операции суперклона, так что эти мультиоперации также принадлежат $\langle R\rangle$. Это понадобится нам при доказательстве дальнейших утверждений. 


\section{Перестановка строк матрицы.}

Перестановка таки двух строк матрицы $X_{g}$, что каждая из них не является последней, порождает матрицу мультиоперации $g^{\prime}$, получающейся из $g$ перестановкой тех аргументов, номера которых совпадают с номерами переставляемых строк матрицы. Следовательно, $g^{\prime} \in\langle R\rangle$.

Если переставим первую и последнюю строки, получим матрицу мультиоперации $(\mu g) \in\langle R\rangle$.

Таким образом, перестановка любых строк матрицы $X_{g}$ дает матрицу мультиоперации, которая получается из $g$ с помощью операций транспозиции, циклической перестановки аргументов и разрешимости.

\section{Удаление строк матрицы.}

Удаление любой строки матрицы $X_{g}$ кроме последней, т. е. удаление $i$-й строки, где $i \in\{1, \ldots, m\}$, дает матрицу мультиоперации

$$
\left(g *_{i}\left(\Delta^{2} \varepsilon\right)\right) \in\langle R\rangle .
$$

Удаление последней строки матрицы $X_{g}$ дает матрицу мультиоперации

$$
\left((\mu(\underbrace{\zeta \ldots \zeta}_{m-1}) \mu g) *_{2}\left(\Delta^{2} \varepsilon\right)\right) \in\langle R\rangle .
$$

\section{Приписывание строк к матрице.}

Покажем, что добавление любой имеющейся строки дает матрицу мультиоперации, которая выражается через $g$ и операции суперклона. Достаточно показать, что если у матрицы $X_{g}$ первую строку записать два раза подряд, то получим матрицу мультиоперации $g^{\prime} \in\langle R\rangle$, которая выражается через $g$ и операции суперклона. Действительно, в этом случае

$$
g^{\prime}=\left(\Delta_{2,3}\left(\left(e_{2}^{2} *_{1} h_{1}\right) *_{3} g\right)\right),
$$

где $h_{1}=e_{2}^{2} *\left(\mu e_{2}^{2}, e_{2}^{2}\right) \in\langle R\rangle$.

\section{Диагонализация матрицы.}

Пусть мультиоперация $g$ представима матрицей $X_{g}$, имеющей вид

$$
\left(\begin{array}{llll}
a_{1}^{1} & a_{1}^{2} & \ldots & a_{1}^{k} \\
a_{2}^{1} & a_{2}^{2} & \ldots & a_{2}^{k} \\
\ldots & \ldots & \ldots & \ldots \\
a_{m+1}^{1} & a_{m+1}^{2} & \ldots & a_{m+1}^{k}
\end{array}\right)
$$

и на множестве $\{1, \ldots, m+1\}$ задано некоторое отношение эквивалентности. Обозначим через $g^{\prime}$ мультиоперацию, у которой матрица состоит из всех таких столбцов матрицы $X_{g}$, что из эквивалентности $i$ и $j$ следует $a_{i}^{s}=a_{j}^{s}$ для любого $s \in\{1, \ldots, k\}$. Будем говорить, что $g^{\prime}$ получена из $g$ с помощью диагонализации. Заметим, что

$$
g^{\prime}=h_{1} *(g, d),
$$

где $h_{1}=e_{2}^{2} *\left(\mu e_{2}^{2}, e_{2}^{2}\right) \in\langle R\rangle$ и $d-$ диагональная мультиоперация для заданного отношения эквивалентности. Как будет показано ниже, любая диагональная мультиоперация принадлежит любому нормализатору. 
Шириной мультиоперации $g \in M_{A}^{m}$ называется число, равное

$$
\sum_{\left(a_{1}, \ldots, a_{m}\right) \in A^{m}}\left|g\left(a_{1}, \ldots, a_{m}\right)\right| .
$$

Назовем $n$-абсииссой матрицу, элементы которой принадлежат $A$, с $n$ столбцами и $k^{n}$ строками, где строки являются всевозможными наборами длины $n$, расположенными в лексикографическом порядке.

Лемма 3. Пусть $K-$ клон и $g$ - произвольная мультиоперачия размерности $n$ и ширины $p$, принадлежащая $N(K)$. Тогда g можно получить из р-универсальной мультиоперации $h$ для $K$ с помощью операций суперклона.

Доказательство. Очевидно, можно считать, что матрица $X_{g}$ не содержит одинаковых строк. Упорядочим лексикографически строки матрицы $X_{g}$. Матрицу $X_{h}$ выпишем так, чтобы первые $p$ столбцов соответствовали проекциям $e_{1}^{p}, \ldots, e_{p}^{p}$. Тогда первые $p$ столбцов матрицы $X_{h}$ образуют $p$-абсциссу. Нетрудно видеть, что все строки матрицы $X_{g}$ содержатся в $p$-абсциссе. Удалим из $X_{h}$ те строки, которые соответствуют строкам $p$-абсциссы, не встречающимся в $X_{g}$. Полученная матрица будет матрицей мультиоперации $g$. Докажем это.

Допустим, что в полученной матрице присутствует столбец $\left(a_{1}, \ldots, a_{n+1}\right)^{t}$, не встречающийся в матрице $X_{g}$. Он соответствует некоторой $f \in K$. Тогда найдутся строки $p$-абсциссы

$$
\left(b_{1}^{i}, \ldots, b_{p}^{i}\right)
$$

где $i \in\{1, \ldots, n+1\}$, для которых $f\left(b_{1}^{i}, \ldots, b_{p}^{i}\right)=a_{i}$. При этом

$$
a_{n+1} \notin g\left(a_{1}, \ldots, a_{n}\right)=g\left(f\left(b_{1}^{1}, \ldots, b_{p}^{1}\right), \ldots, f\left(b_{1}^{n}, \ldots, b_{p}^{n}\right)\right) .
$$

С другой стороны, $b_{j}^{n+1} \in g\left(b_{j}^{1}, \ldots, b_{j}^{n}\right)$, где $j \in\{1, \ldots, p\}$. Значит,

$$
a_{n+1} \in f\left(g\left(b_{1}^{1}, \ldots, b_{1}^{n}\right), \ldots, g\left(b_{p}^{1}, \ldots, b_{p}^{n}\right)\right),
$$

что противоречит условию $g \in N(K)$. Таким образом, полученная матрица является матрицей мультиоперации $g$.

$$
\begin{gathered}
\text { Пусть } f \in P_{A}^{n} \text { и матрица } Y=\left(\begin{array}{cccc}
b_{1}^{1} & b_{1}^{2} & \ldots & b_{1}^{n} \\
b_{2}^{1} & b_{2}^{2} & \ldots & b_{2}^{n} \\
\ldots & \ldots & \ldots & \ldots \\
b_{s}^{1} & b_{s}^{2} & \ldots & b_{s}^{n}
\end{array}\right) . \text { Тогда } \\
f(Y)=\left(\begin{array}{c}
f\left(b_{1}^{1}, b_{1}^{2}, \ldots, b_{1}^{n}\right) \\
f\left(b_{2}^{1}, b_{2}^{2}, \ldots, b_{2}^{n}\right) \\
f\left(b_{s}^{1}, b_{s}^{2}, \ldots, b_{s}^{n}\right)
\end{array}\right) .
\end{gathered}
$$

Матрица $X$ называется $g$-матрицей, если любой столбец матрицы $X$ является столбцом матрицы $X_{g}$.

Пусть размерность мультиоперации $g$ равна $m$ и матрица $X_{g}$ имеет вид 


$$
\left(\begin{array}{llll}
a_{1}^{1} & a_{1}^{2} & \ldots & a_{1}^{k} \\
a_{2}^{1} & a_{2}^{2} & \ldots & a_{2}^{k} \\
\ldots & \ldots & \ldots & \ldots \\
a_{m+1}^{1} & a_{m+1}^{2} & \ldots & a_{m+1}^{k}
\end{array}\right)
$$

Тогда верно следующее утверждение.

Лемма 4. Для $f \in P_{A}^{n} u g \in M_{A}^{m}$ полутождество перестановочности выполняется тогда и только тогда, когда для любой g-матриць $X$ существует такое $i \in\{1, \ldots, k\}$, чmо $f(X)=\left(a_{1}^{i}, \ldots, a_{m+1}^{i}\right)^{t}$.

Доказательство. Необходимость. Предположим противное. Пусть

$$
X=\left(\begin{array}{llll}
b_{1}^{1} & b_{1}^{2} & \cdots & b_{1}^{n} \\
b_{2}^{1} & b_{2}^{2} & \cdots & b_{2}^{n} \\
\cdots & \cdots & \cdots & \cdots \\
b_{m+1}^{1} & b_{m+1}^{2} & \cdots & b_{m+1}^{n}
\end{array}\right)
$$

и $f(X)=\left(c_{1}, \ldots, c_{m+1}\right)^{t}$, где $\left(c_{1}, \ldots, c_{m+1}\right)^{t}$ не является столбцом матрицы $X_{g}$. Тогда

$$
f *\left(g\left(b_{1}^{1}, \ldots, b_{m}^{1}\right), \ldots, g\left(b_{1}^{n}, \ldots, b_{m}^{n}\right)\right) \not \subseteq g *\left(f\left(b_{1}^{1}, \ldots, b_{1}^{n}\right), \ldots, f\left(b_{m}^{1}, \ldots, b_{m}^{n}\right)\right),
$$

так как $c_{m+1}=f\left(b_{m+1}^{1}, \ldots, b_{m+1}^{n}\right) \in f *\left(g\left(b_{1}^{1}, \ldots, b_{m}^{1}\right), \ldots, g\left(b_{1}^{n}, \ldots, b_{m}^{n}\right)\right)$ и $c_{m+1} \notin$ $g\left(c_{1}, \ldots, c_{m}\right)=g *\left(f\left(b_{1}^{1}, \ldots, b_{1}^{n}\right), \ldots, f\left(b_{m}^{1}, \ldots, b_{m}^{n}\right)\right)$. Противоречие.

Достаточность. Мы должны показать, что для любых элементов $b_{1}^{1}, \ldots, b_{m}^{1}, \ldots, b_{1}^{n}, \ldots, b_{m}^{n}$ из $A$ выполняется включение

$$
f *\left(g\left(b_{1}^{1}, \ldots, b_{m}^{1}\right), \ldots, g\left(b_{1}^{n}, \ldots, b_{m}^{n}\right)\right) \subseteq g *\left(f\left(b_{1}^{1}, \ldots, b_{1}^{n}\right), \ldots, f\left(b_{m}^{1}, \ldots, b_{m}^{n}\right)\right) .
$$

Если $\left(\begin{array}{cccc}b_{1}^{1} & b_{1}^{2} & \ldots & b_{1}^{n} \\ b_{2}^{1} & b_{2}^{2} & \ldots & b_{2}^{n} \\ \ldots & \ldots & \ldots & \ldots \\ b_{m}^{1} & b_{m}^{2} & \ldots & b_{m}^{n}\end{array}\right)$ не равна некоторой $g$-матрице без последней строки, то $f *\left(g\left(b_{1}^{1}, \ldots, b_{m}^{1}\right), \ldots, g\left(b_{1}^{n}, \ldots, b_{m}^{n}\right)\right)=\varnothing$, а значит, включение верно.

Итак, пусть $\left(\begin{array}{cccc}b_{1}^{1} & b_{1}^{2} & \ldots & b_{1}^{n} \\ b_{2}^{1} & b_{2}^{2} & \ldots & b_{2}^{n} \\ \ldots & \ldots & \ldots & \ldots \\ b_{m}^{1} & b_{m}^{2} & \ldots & b_{m}^{n}\end{array}\right)$ равна некоторой $g$-матрице без последней строки и существует такой элемент $a$, что

$$
a \in f *\left(g\left(b_{1}^{1}, \ldots, b_{m}^{1}\right), \ldots, g\left(b_{1}^{n}, \ldots, b_{m}^{n}\right)\right)
$$

и

$$
a \notin g *\left(f\left(b_{1}^{1}, \ldots, b_{1}^{n}\right), \ldots, f\left(b_{m}^{1}, \ldots, b_{m}^{n}\right)\right) .
$$

Из включения $a \in f *\left(g\left(b_{1}^{1}, \ldots, b_{m}^{1}\right), \ldots, g\left(b_{1}^{n}, \ldots, b_{m}^{n}\right)\right)$ следует, что $a=$ $f\left(b^{1}, \ldots, b^{n}\right)$, где $b^{j} \in g\left(b_{1}^{j}, \ldots, b_{m}^{j}\right)$. 
Очевидно, что $Z=\left(\begin{array}{cccc}b_{1}^{1} & b_{1}^{2} & \ldots & b_{1}^{n} \\ b_{2}^{1} & b_{2}^{2} & \ldots & b_{2}^{n} \\ \ldots & \ldots & \ldots & \ldots \\ b_{m}^{1} & b_{m}^{2} & \ldots & b_{m}^{n} \\ b^{1} & b^{2} & \ldots & b^{n}\end{array}\right)$ является $g$-матрицей. Тогда

$$
f(Z)=\left(\begin{array}{c}
a_{1}^{i} \\
a_{2}^{i} \\
\cdots \\
a_{m}^{i} \\
a_{m+1}^{i}
\end{array}\right)
$$

для некоторого $i \in\{1, \ldots, k\}$.

Отсюда следует, что $a=a_{m+1}^{i}$ и $a \in g\left(a_{1}^{i}, \ldots, a_{m}^{i}\right)$, т. е.

$$
a \in g *\left(f\left(b_{1}^{1}, \ldots, b_{1}^{n}\right), \ldots, f\left(b_{m}^{1}, \ldots, b_{m}^{n}\right)\right) .
$$

Противоречие.

Следствие 1. Любой нормализатор содержит все диагональные мультиопераиии.

Доказательство. В силу определения диагональных мультиопераций и леммы 4 каждая диагональная мультиоперация нормальна относительно любой операции. Следовательно, все диагональные мультиоперации должны принадлежать любому нормализатору.

Декартовым произведением матриц $X_{1}$ и $X_{2}$ называется матрица

$$
X_{1} \times X_{2}
$$

состоящая из всевозможных столбцов вида $\left(a_{1}, \ldots, a_{k}, b_{1}, \ldots, b_{s}\right)^{t}$, где $\left(a_{1}, \ldots, a_{k}\right)^{t}-$ столбец $X_{1}$ и $\left(b_{1}, \ldots, b_{s}\right)^{t}-$ столбец $X_{2}$.

Пусть $g_{1} \in M_{A}^{n}$ и $g_{2} \in M_{A}^{m}$. Нетрудно видеть, что мультиоперация

$$
g=\left(e_{2}^{2} *_{1}\left(\zeta \mu\left(e_{2}^{2} *_{2} g_{1}\right)\right)\right) *_{n+2} g_{2}
$$

имеет матрицу $X_{g_{1}} \times X_{g_{2}}$, причем если $g_{1}, g_{2} \in\langle R\rangle$, то $g \in\langle R\rangle$.

Обозначим через $X^{n}$ матрицу $\underbrace{X \times \ldots \times X}_{n}$.

Лемма 5. Для любых $g_{1}, g_{2} \in\langle R\rangle$ существует такая мультиоперащия $g \in\langle R\rangle$, что $S(g)=S\left(g_{1}\right) \cap S\left(g_{2}\right)$.

Доказательство. Если у одной из мультиопераций, например $g_{1}$, значения на всех наборах равны пустому множеству, то очевидно, что в качестве $g$ нужно взять мультиоперацию $g_{2}$. Пусть теперь ни одна из мультиопераций $g_{1}$ и $g_{2}$ не является мультиоперацией, у которой значения на всех наборах равны пустому множеству. Докажем, что если в качестве $g$ взять мультиоперацию, матрица которой равна $X_{g_{1}} \times X_{g_{2}}$, то $S(g)=S\left(g_{1}\right) \cap S\left(g_{2}\right)$. Вначале отметим, что $S\left(g_{1}\right) \cap S\left(g_{2}\right)=S\left(\left\{g_{1}, g_{2}\right\}\right)$. 
Включение $S\left(\left\{g_{1}, g_{2}\right\}\right) \subseteq S(g)$ выполняется по предложению 1 , так как из $g_{1}, g_{2} \in$ $N\left(S\left(\left\{g_{1}, g_{2}\right\}\right)\right)$ следует $g \in N\left(S\left(\left\{g_{1}, g_{2}\right\}\right)\right)$.

Покажем, что $S(g) \subseteq S\left(\left\{g_{1}, g_{2}\right\}\right)$. Для этого достаточно доказать, что $g_{1}$ и $g_{2}$ выражаются с помощью $g$ и операций суперклона. Действительно, для получения матрицы мультиоперации $g_{1}$ удаляем первые $n+1$ строк матрицы $X_{g_{1}} \times X_{g_{2}}$, а для получения матрицы мультиоперации $g_{2}$ удаляем последние $m+1$ строк. Удаление строк матрицы, как было показано ранее, не выводит за границы суперклона.

Пусть $g_{1}, g_{2} \in M_{A}^{m}$. Если для любых $a_{1}, \ldots, a_{m}$ из $A$ выполняется включение

$$
g_{1}\left(a_{1}, \ldots, a_{m}\right) \subseteq g_{2}\left(a_{1}, \ldots, a_{m}\right),
$$

то будем писать $g_{1} \leqslant g_{2}$.

Пересечением $g_{1}$ и $g_{2}$ называется такая мультиоперация $g_{1} \cap g_{2}$, что для любых $a_{1}, \ldots, a_{m}$ из $A$

$$
\left(g_{1} \cap g_{2}\right)\left(a_{1}, \ldots, a_{m}\right)=g_{1}\left(a_{1}, \ldots, a_{m}\right) \cap g_{2}\left(a_{1}, \ldots, a_{m}\right) .
$$

Заметим, что если $g_{1}, g_{2} \in\langle R\rangle$, то $g_{1} \cap g_{2}=\left(e_{2}^{2} *\left(\mu e_{2}^{2}, e_{2}^{2}\right)\right) *\left(g_{1}, g_{2}\right) \in\langle R\rangle$.

Доказательство теоремы 2. Покажем сначала, что $\langle R\rangle \subseteq N(S(R))$.

Пусть $g \in R$. Тогда $g$ нормальна относительно всех $f \in S(R)$, а значит, $g \in N(S(R))$. Следовательно, $R \subseteq N(S(R))$ и $\langle R\rangle \subseteq\langle N(S(R))\rangle$. Так как по предложению 1 любой нормализатор является суперклоном, то $\langle N(S(R))\rangle=N(S(R))$.

Теперь докажем, что $N(S(R)) \subseteq\langle R\rangle$. В силу включения $N(S(R)) \subseteq N(S(\langle R\rangle))$ достаточно будет показать, что $N(S(\langle R\rangle)) \subseteq\langle R\rangle$.

Вначале рассмотрим случай, когда $\langle R\rangle$ является конечнопорожденной алгеброй. Обозначим через $\left\{g_{1}, \ldots, g_{s}\right\}$ множество образующих $\langle R\rangle$. Тогда

$$
S(\langle R\rangle)=S\left(\left\{g_{1}, \ldots, g_{s}\right\}\right)=\bigcap_{i=1}^{s} S\left(g_{i}\right) .
$$

Согласно лемме 5 найдется такая операция $g \in\langle R\rangle$, что $S(\langle R\rangle)=S(g)$. Если мы покажем, что из $g$ с помощью операций суперклона для любого $n$ можно получить $n$-универсальную мультиоперацию $h$, то с учетом леммы 3 требуемое включение $N(S(\langle R\rangle)) \subseteq\langle R\rangle$ будет доказано.

В [6] предложен метод построения так называемого $n$-графика. Лемма 4 дает возможность использовать этот метод для построении $n$-универсальной мультиоперации $h$ при произвольном $n$. Приведем изложение данного метода применительно к $n$-универсальной мультиоперации $h$.

Пусть $g$ - мультиоперация размерности $m$ и ширины $p$, а $X_{1}, \ldots, X_{l}$ - всевозможные $g$-матрицы размера $(m+1) \times n$, где $l=p^{n}$. Построим матрицу $X$, записав $X_{1}, \ldots, X_{l}$ друг под другом:

$$
X=\left(\begin{array}{c}
X_{1} \\
X_{2} \\
\ldots \\
X_{l}
\end{array}\right) .
$$

Легко видеть, что для любой операции $f$ размерности $n$, стабильной относительно $g, f(X)$ является столбцом матрицы $X_{g}^{l}$. Из матрицы $X_{g}^{l}$ мы и получим матрицу 
для $h$. Мультиоперация, представимая матрицей $X_{g}^{l}$, как известно, принадлежит $\langle R\rangle$. Матрица $X$ имеет вспомогательный характер, подсказывая необходимые действия над $X_{g}^{l}$, т. е. все преобразования матриц $X$ и $X_{g}^{l}$ будут проводиться параллельно. В конечном итоге мы должны из матрицы $X$ получить $n$-абсциссу, а $X_{g}^{l}$ станет матрицей, в которой столбцы представляют все $n$-местные операции из $S(\langle R\rangle)$, т. е. матрицей $h$.

Итак, во-первых, проведем диагонализацию матрицы $X_{g}^{l}$. Соответствующее отношение эквивалентности на множестве $\{1, \ldots, m l\}$ зададим следующим образом: $i$ и $j$ эквивалентны, если $i$-я и $j$-я строки матрицы $X$ равны.

После диагонализации для матриц $X$ и $X_{g}^{l}$ будет выполняться следующее условие: если две строки матрицы $X$ равны, то строки матрицы $X_{g}^{l}$ с такими же номерами также равны.

Далее, удалением строк в матрице $X$ добьемся, чтобы в ней не было одинаковых строк. Соответствующие строки удалим и в матрице $X_{g}^{l}$.

Теперь пара $\left(X, X_{g}^{l}\right)$ уже определяет все $n$-местные операции из $S(\langle R\rangle)$. Строки матрицы $X$ являются наборами длины $n$, а столбцы матрицы $X_{g}^{l}$ задают значения всех $n$-местных операций на этих наборах. Отметим, что среди строк матрицы $X$ могут быть не все наборы длины $n$; очевидно, на таких наборах значения операций являются произвольными.

Учитывая этот факт, завершим преобразования матриц $X$ и $X_{g}^{l}$ следующим образом. Добавим в $X$ отсутствующие строки так, чтобы $X$ содержала все строки $n$-абсциссы. Если их количество равно $r$, то новую матрицу $X_{g}^{l}$ получим декартовым произведением матрицы $X_{g}^{l}$ на матрицу $Z$, где $Z$ состоит из всевозможных столбцов длины $r$. Отметим, что матрица $Z$ представляет мультиоперацию $g_{z}$ размерности $r-1$, которая на любом наборе принимает значение $A$. Очевидно, что $g_{z}$ принадлежит любому суперклону. Отсюда следует, что новая матрица $X_{g}^{l}$ представляет мультиоперацию, принадлежащую $\langle R\rangle$.

Осталось переставить строки матриц $X$ и $X_{g}^{l}$ так, чтобы матрица $X$ стала $n$-абсциссой. Теперь $X_{g}^{l}$ представляет $n$-универсальную мультиоперацию $h$.

Для случая конечнопорожденной алгебры $\langle R\rangle$ доказательство завершено.

Рассмотрим случай, когда суперклон $\langle R\rangle$ имеет бесконечное множество образующих $\left\{g_{1}, \ldots, g_{s}, \ldots\right\}$. Тогда

$$
S(\langle R\rangle)=\bigcap_{i=1}^{\infty} S\left(g_{i}\right) .
$$

Обозначим через $R_{j}$ суперклон, порожденный конечным множеством образующих $\left\{g_{1}, \ldots, g_{j}\right\}$, и $S_{j}=\bigcap_{i=1}^{j} S\left(g_{i}\right)$. Отсюда $S_{1} \supseteq S_{2} \supseteq \ldots$ и

$$
S(\langle R\rangle)=\bigcap_{j=1}^{\infty} S_{j} .
$$

Нетрудно заметить, что $\langle R\rangle=\bigcup_{j=1}^{\infty} R_{j}$ и $n$-универсальная мультиоперация $h_{j}$ для $S_{j}$ принадлежит $R_{j}$, а значит, $h_{j} \in\langle R\rangle$. 
Далее имеем

$$
h_{1} \geqslant h_{2} \geqslant \ldots \geqslant h_{j} \geqslant \ldots, \quad h=\bigcap_{j=1}^{\infty} h_{j} .
$$

Для любого $n$ размерность $n$-универсальной мультиоперации $h_{j}$ равна $k^{n}-1$, где $k-$ мощность $A$. Очевидно, таких мультиопераций конечное число, поэтому убывающая последовательность (1) стабилизируется для некоторого $N$, которое зависит только от $n$. Отсюда следует, что $h=\bigcap_{j=1}^{N} h_{j}$, а значит, $h \in\langle R\rangle$. Теорема доказана.

Следствие 2. Отображения $S(x)$ и $N(x)$ являются взаимно обратными антиизоморфизмами решетки клонов и решетки суперклонов.

Таким образом, отображения $S(x)$ и $N(x)$ определяют совершенную связь Галуа [12] между клонами и суперклонами.

Стабилизатор множества операций называют централизатором. При этом вместо полутождества перестановочности получается тождество перестановочности $[3,4]$. Отметим, что централизатор определяет на множестве клонов двойственное себе соответствие Галуа [12], которое не является совершенным. Но если вместо алгебраического замыкания взять параметрическое замыкание [13], то отображение, задаваемое централизатором, будет определять совершенную связь Галуа для решетки параметрически замкнутых множеств операций [13].

\section{Список литературы}

1. Яблонский С. В., “О суперпозициях функций алгебры логики”, Матем. сб., 30 (1952), 329-348.

2. Яблонский С. В., "Функциональные построения в $k$-значной логике", Труды Матем. ин-та им. В.А.Стеклова АН СССР, 51 (1958), 5-142.

3. Мальцев А. И., "Итеративные алгебры и многообразия Поста", Алгебра и логика, 1966, № $5,3-9$.

4. Кон П., Универсальная алгебра, М.: МИР, 1968, 352 с.

5. Lau D., Function algebras on finite sets. A basic course on many-valued logic and clone theory, Springer-Verlag, Berlin, 2006, 668 c.

6. Боднарчук В. Г., Калужнин Л. А., Котов В.Н., Ромов Б. А., "Теория Галуа для алгебр Поста", Кибернетика, 1969, № 3, С. 1-10; № 5, 1-9..

7. Poscher R., Kaluznin L. A., Funktionen- und Relationenalgebren, Ein Kapitel der diskreten Mathematik, VEB DVW, Berlin, 1979, 237 pp.

8. Перязев Н. А., "Недоопределенные частичные булевы функции", Матер. XV Междунар. конф. «Проблемы теоретической кибернетики», 2008, 92.

9. Перязев Н. А., "Клоны, ко-клоны, гиперклоны и суперклоны", Ученые записки Казанского гос. ун-та. Серия: Физико-матем. науки, 151:2 (2009), 120-125.

10. Перязев Н. А., "Мультиоперации и суперклоны”, Междунар. конф. «Мальцевские чтения». Тезисы докл., 2011, 84.

11. Перязев Н. А., “Теория Галуа для клонов и суперклонов”, Материалы XVI Междунар. конф. «Проблемы теоретической кибернетики», 2011, 359-363.

12. Оре О., Теория графов, Наука, Москва, 1980, 336 с.

13. Кузнецов А. В., "О средствах для обнаружения невыводимости или невыразимости", В кн., Логический вывод, Наука, Москва, 1979, 5-33. 